\title{
Serum Visfatin Levels Estimated in Overweight Individuals
}

\author{
*F Kabir ${ }^{1}$, SA Haque 2 , KMG Haque 3
}

\begin{abstract}
Background: Visfatin is a novel adipocytokine predominantly secreted from visceral fat and its blood shows insulin-mimetic effects which correlate with overweight, type 2 diabetes mellitus (T2DM) and inflammation. Overweight is an important component of metabolic syndrome. However, the relationship between visfatin levels and overweight is not yet clear. The aim of this study was to investigate the relationship of serum visfatin levels in overweight and apparently healthy subjects.

Methodology: This was a cross-sectional comparative study includes 50 overweight subjects who had no disease conditions and 42 controls. Anthropometric measurements included Height (HT), body mass index (BMI), waist circumferences (WC) and hip circumferences (HC) were performed by using standard methods. Serum visfatin levels were analyzed by Enzyme Linked Immunosorbent Assay (ELISA). Biochemical parameters estimated by ELISA and standard methods where fasting serum visfatin, fasting serum insulin and fasting plasma glucose, serum triglycerideg (TG), serum cholesterol (CHOL) levels and lipid profiles were evaluated. All data were presented as Mean $(\mathrm{M})$ and Standard Deviation $( \pm \mathrm{SD})$ and results were compared with students t-test and correlation coefficient. The values were considered significant at 0.05 level of significance.
\end{abstract}

Results: BMI, WC, $\mathrm{HC}$ and \%BFM were significantly higher in overweight subjects compaired to controls. Serum visfatin $(\mathrm{ng} / \mathrm{ml})$ value in overweight subjects also was observed significantly higher compared to the controls.

Conclusion: A significant corelation of plasma visfatin levels was observed with anthropometric parameters whereas no significant relationship observed with any of the biochemical parametres either the study subjects or controls. This study shows correlation between serum visfatin and overweight subjects. Future studies with large population possibly will explore and explain the role and relationship of serum visfatin with overweight, T2DM and others diseases as related to visfatin.

Key Words: Visfatin, overweight, BMI, lipid profiles.

\section{Introduction}

Overweight is one of the prevailing diseases in the present which is described as accumulation of adipose tissues. In the past, adipose tissue was considered as storehouse of fats and energy but recent reports redefined it as a major endocrine organ that secretes adipocytokines. ${ }^{1}$ Adipocytokines, secreted from adipose tissues which play an important role in regulation of food intake and body weight, insulin sensitivity, immunity, inflammation and vascular haemostasis. ${ }^{2,3,4}$ Adipocytokines are

thought to be a missing link between T2DM and insulin resistance, the mechanism in which it induces insulin resistance and its role in the pathogenesis of T2DM has not yet been clarified. ${ }^{5}$ In 2005, Fukuhara et al first described visfatin is one of the adipocytokines which predominantly produced by visceral adipose tissue and found in liver, muscle, bone marrow, lung, heart, placenta, kidney tissue and peripheral lymphocytes. ${ }^{6}$ It is noticed that administration of visfatin in mice lowered both

$1 *$ Dr. Fahmida Kabir, Associate Professor of Biochemistry, Green Life Medical College, Dhaka

${ }^{2}$ Dr. Shiekh Anisul Haque, Lecturer of Bio-Chemistry, Green Life Medical College, Dhaka

${ }^{3} \mathrm{Dr}$. KMG Haque, KFSH, Dammam, KSA

*Corresponding Author

Date of submission: 10.10.2017, Date of acceptance: 14.01.2018

AKMMC J 2018; 9(2) : 91-95 
glucose and insulin levels, whereas plasma glucose levels was shown higher in mice heterozygous for visfatin deficiency compared to it in normal mice. ${ }^{7,8}$ Published data also suggest that visfatin had effects on lipid homeostasis similar to that of insulin and it is responsible for adipocyte proliferation, differentiation and triglyceride (TG) metabolism. ${ }^{9}$ Based on these findings, circulating visfatin levels and its determinants have been investigated in a number of clinical studies $6,10,11,12$ that observed visfatin expression in visceral fats is increased in overweight subjects and plasma levels of visfatin associated to a great extent with amount of visceral fat than that of adipose tissues. Overweight and T2DM are emerging as central health problems worldwide. Rise in overweight is associated to a subsequent increase in risk of T2DM. The International Association for the Study of Obesity/International Obesity Taskforce analysis in the year 2010 estimates that approximately one billion adults are currently overweight (Body Mass Index [BMI] 25- 29.9 $\mathrm{Kg} / \mathrm{m}^{2}$ ), and a further 475 million adults are obese. With an Asian-specific definition of obesity $\left(\mathrm{BMI}>28 \mathrm{Kg} / \mathrm{m}^{2}\right)$, global obesity prevalence in adults is estimated at $>600$ million. ${ }^{13,14}$ It has been reported that it is primarily central or visceral fat relatively than subcutaneous fat seen in the region of big buttocks and thighs which is responsible for unfavorable ramifications of overweight. ${ }^{15}$ This feature has drawn particular attention by raising question about potential differences between visceral and subcutaneous fat. Reviewing above findings, it may be suggested that visfatin might serve as a new link between overweight and T2DM but in an outwardly inconsistent manner. Furthermore, observational studies examined association of visfatin with adiposity and differential regional fat distribution have shown great discrepancies in their findings and more study is needed to establish the role of visfatin in visceral overweight. This crosssectional comparative study was carried out in a private multi purpose specialized hospital which is situated in central Dhaka, from July 2007 and July 2008. A recent study in Bangladeshi population observed elevated plasma visfatin level in T2DM.16 Aim of this study was to examine the relationship of visfatin and overweight by analyzing biochemical and anthropometric parameters in overweight individuals and apparently normal healthy adults.

\section{Mathodology \\ Informed consent}

All protocols used for the recruitment of patients / healthy subjects and the collection of blood samples was reviewed and approved by the appropriate competent committee.

\section{Study populations \\ Healthy individuals, Group-I (Gp-I)}

Forty two age- (41 6 years, Mean \pm SD), sex- and BMI-matched apparently healthy adult volunteers who had no family history of T2DM were recruited as Controls.

\section{Overweight subjects Group-II (Gp-II)}

Fifty adults, age 436 (Mean \pm SD) years who attended our Out-Patients Clinic seeking consultation for simple overweight were investigated in this study. All those 50 individuals were confirmed by clinical and laboratory examination that they are free from T2DM or any other known diseases. There was no specific predilection for race, religion and socioeconomic status.

\section{Anthropometric measurements}

Anthropometric measurements including HT in meter $(\mathrm{m}), \mathrm{BW}$ in kilograms $(\mathrm{kg})$, WC in centimeter $(\mathrm{cm}), \mathrm{HC}$ in $\mathrm{cm}$, were measured by applying appropriate standard techniques. BMI of the subjects were calculated using international standard formula, BMI $=$ Weight $(\mathrm{Kg}) /[\text { Height }(\mathrm{m})]^{2}$. Simple overweight was defined when an overweight individual shows $\mathrm{BMI}^{17}$ between $25-29 \mathrm{Kg} / \mathrm{m}^{2}$. Percentage BFM was measured by Omron Body Fat Monitor (Omron Corporation, Japan) following manufacturer instructions.

\section{Biochemical Parameters}

Serum Glucose and Lipid profiles were measured by enzymatic colorimetric method (Randox Laboratories, UK) and estimation was carried out in an autoanalyzer (Hitachi 704). Insulin and Visfatin were estimated by ELISA (kits from Linco Research, and ALPCO diagnostics, USA).

\section{Satistical Analysis}

Comparisons of anthropometric and biochemical characteristics between two groups were analyzed 
using unpaired student's ' $\mathrm{t}$ ' tests. Pearson's correlation analysis were performed to investigate the possible associations between serum concentration of visfatin with anthropometric parameters. The SPSS version 10.0 was used for computing all analyzes.

\section{Results}

Age $(\mathrm{M} \pm \mathrm{SD})$ in Gp-I and Gp-II was 416 and 436 years respectively as shown in Table 1. Mean BMI $(\mathrm{M} \pm \mathrm{SD})$ in $\mathrm{Gp}$-II was significantly higher compared to $\mathrm{Gp}-\mathrm{I}(\mathrm{p}=<0.000)$. WC $(\mathrm{M} \pm \mathrm{SD}), \mathrm{HC}$ $(\mathrm{M} \pm \mathrm{SD}), \% \mathrm{BFM}$ were $81.0 \pm 7.3,89.3 \pm 5.8$ $23.5 \pm 5$ in Gp-I and 93.5 $\pm 7.7,102.2 \pm 7.3$, 32.86 .0 in Gp-II respectively and the difference between two groups is highly significant as shown in Table 2. Fasting serum $\mathrm{TG}(\mathrm{M} \pm \mathrm{SD})$, total $\mathrm{CHOL}(\mathrm{M} \pm \mathrm{SD})$, HDL-CHOL $(\mathrm{M} \pm \mathrm{SD})$ and LDL$\mathrm{CHOL}(\mathrm{M} \pm \mathrm{SD})$ were $163.2 \pm 8.3$, 2o $2 \pm 53$, $35.3 \pm 5,136.7 \pm 49.3$ in Gp-I and 168.5 \pm 7.0 , $207 \pm 43,34.7 \pm 4,139.6 \pm 43$ in Gp-II respectively showed no significant difference between two groups (Table 2). No difference also was observed between Gp-I, $11.2 \pm 4.1(\mathrm{M} \pm \mathrm{SD})$ and Gp-II $11.6 \pm 1.3(\mathrm{M} \pm \mathrm{SD})$ for fasting insulin levels $(\mu \mathrm{U} / \mathrm{ml})$ as shown in Table 3. Fasting serum visfatin $(\mathrm{ng} / \mathrm{ml})$ value in $\mathrm{Gp}$-I and $\mathrm{Gp}$-II was 4.2 4.0 and 7.44 .2 respectively which was significantly higher $(\mathrm{p}=0.000)$ in Gp-II as compared to Gp-I (Table 2). Interestingly, levels of visfatin did not show any significant correlation with fasting glucose, fasting serum insulin, $\mathrm{WC}, \mathrm{HC}, \% \mathrm{BFM}$, TG and Cholesterol.

Table-I: Shows comparison of mean values of anthropometric parameters between Gp-I and Gp-II using unpaired students ' $t$ ' tests of the study subjects $\mathrm{n}=$ number of subjects

\begin{tabular}{lccc}
\hline Variables & Gp-I $(\mathbf{n = 4 2})$ & Gp-II $(\mathbf{n}=\mathbf{5 0})$ & $\mathbf{~} \mathbf{\text { Gp-I versus Gp-II }}$ \\
\hline Age $(\mathrm{Yrs})$ & $42 \pm 5$ & $43 \pm 7$ & 0.125 \\
BMI $\left(\mathrm{Kg} / \mathrm{m}^{2}\right)$ & $20.8 \pm 1.9$ & $28.4 \pm 3.5$ & 0.00 \\
WC & $81.0 \pm 7.3$ & $93.5 \pm 7.7$ & 0.00 \\
HC & $89.3 \pm 5.8$ & $102.2 \pm 7.3$ & 0.00 \\
\% $\mathrm{BFM}$ & $23.5 \pm 5$ & $32.8 \pm 6.0$ & 0.00 \\
\hline
\end{tabular}

Table-II: Shows comparison of mean values of biochemical parameters between Gp-I and Gp-II using unpaired students ' $t$ ' tests of the study subjects. ( $\mathrm{n}=$ number of subjects)

\begin{tabular}{lccc}
\hline & & & \multicolumn{2}{c}{ p values } \\
\cline { 4 - 4 } Variables & Gp-I (n=42) & Gp-II (n=50) & Gp-I versus Gp-II \\
\hline TG (mg/dl) & $163.2 \pm 8.3$ & $168.5 \pm 7.0$ & .921 \\
CHOL (mg/dl) & $202 \pm 53$ & $207 \pm 43$ & .492 \\
$\begin{array}{l}\text { HDL-CHOL } \\
\text { (mg/dl) }\end{array}$ & $35.3 \pm 5$ & $34.7 \pm 4$ & .126 \\
$\begin{array}{l}\text { LDL-CHOL } \\
(\mathrm{mg} / \mathrm{dl})\end{array}$ & $136.7 \pm 49.3$ & $139.6 \pm 43.9$ & .722 \\
$\begin{array}{l}\text { Fasting Glucose } \\
(\mathrm{m} \text { mol-1) }\end{array}$ & $5.2 \pm 1.8$ & $5.9 \pm 1.3$ & .869 \\
$\begin{array}{l}\text { Plasma insulin } \\
(\mu \mathrm{U} / \mathrm{ml})\end{array}$ & $11.2 \pm 4.1$ & $11.6 \pm 1.3$ & .123 \\
$\begin{array}{l}\text { Serum visfatin } \\
(\mathrm{ng} / \mathrm{ml})\end{array}$ & $4.2 \pm 4.0$ & $7.4 \pm 4.2$ & .002 \\
\hline
\end{tabular}

Table-III: Shows correlation of plasma visfatin level with biochemical and anthropometric parameters. Data are presented as $\mathrm{M} \pm \mathrm{SD}, \mathrm{P}<0.05$ and Correlation Coefficient (r2).

\begin{tabular}{lcccc}
\hline \multirow{2}{*}{ Variables } & \multicolumn{2}{c}{ Gp-I $(\mathbf{n}=\mathbf{4 2})$} & \multicolumn{2}{c}{ Gp-I $(\mathbf{n}=\mathbf{4 2})$} \\
\cline { 2 - 5 } BMI & $\mathrm{R}$ & $\mathrm{P}$ & $\mathrm{R}$ & $\mathrm{P}$ \\
WC & .069 & 0.679 & 0.082 & 0.251 \\
HC & 0.082 & 0.624 & 0.082 & 0.251 \\
\%BFM & 0.078 & & 0.118 & 0.420 \\
Insulin & 0.067 & 0.640 & 0.078 & 0.593 \\
Fasting Glucose & 0.047 & 0.781 & 0.049 & 0.740 \\
TG & 0.163 & 0.940 & 0.065 & 0.657 \\
Cholesterol & 0.167 & 0.126 & 0.156 & 0.283 \\
\hline
\end{tabular}

\section{Discussion}

Overweight specific adipocytokines increased levels could play a vital role in obese associated metabolic derangements. ${ }^{18}$ Our study is intended to investigate serum levels of visfatin and its correlation to nondiabetic overweight individuals. The increasing prevalence of overweight around the globe is becoming an important public health concern in adulthood and presents various complications. Because visceral adipose tissue is considered as an important source of visfatin, studies on visfatin alterations in adults may be useful in understanding 
some of the complications of overweight. This study shows a significant higher serum visfatin in Gp-II than Gp-I which supports the findings by Haider et $a l^{10}$ whereas Pagano et $a l^{19}$ observed plasma visfatin was significantly lower in control subjects than overweight individuals. Visfatin is released not only by adipose tissue but also by peripheral blood mononuclear cells as pre-B cell colony-enhancing factors. ${ }^{20,21}$ Therefore, subclinical inflammation in overweight may have enhanced expression of proinflammatory cytokines including visfatin expression factor. A significant correlation between visfatin concentrations and WC found by others 22,23 this is in observance with the fact that visfatin is secreted preferably from visceral fats rather than subcutaneous ones as described by Fukuhara et al6 and Berdent et al. ${ }^{24}$ This study observed significant correlation between serum visfatin and $\mathrm{WC}, \mathrm{HC}$, BMI and \% BFM postulates that amount of body fat possibly influencing the concentration of circulating visfatin in overweight. Furthermore, Chen et al 21 observed visfatin level was significantly associated with total CHOL, TG, HDL-CHOL, and LDL-CHOL in simple re-regression analysis but not in multiple regression. However, this study shown no significant correlation between serum visfatin and total CHOL, TG, HDL-CHOL, LDLCHOL levels. It is still indistinguishable whether visfatin influences lipid profile limits or vice versa. We propose that visfatin elevations are independent to glucose, insulin and insulin resistance, which indicate that elevated visfatin levels are related to other factors like visceral fat deposit and inflammation. Fahmida et $a l^{16}$ reported that serum visfatin levels are independent and significantly associated with T2DM. Our report also is in consistent with earlier report who observed no correlation between visfatin levels and fasting plasma glucose. We like to hpothesize that visfatin is more plausibly to be a marker of overweight as a product of visceral fat cells rather than a new key in the pathogenesis of T2DM. The absence of a relationship with biochemical parameters suggest visfatin is independently regulated in overweight subjects. These findings are new in association with the role of visfatin on overweight. We wish to test this hypothesis in a well-designed large population study to clarify these divisive data on visfatin and uncover relationship of adipocytokines to overweight, insulin resistance T2DM and systemic metabolic syndromes.

Conflict of interests: There is no conflict of interests

\section{Acknowledgements}

The authors thank all volunteers who participated and co-operated in the study. We gratefully acknowledged the support of our research fellows and technical staff for their kind co-operation and help.

\section{References}

1. Bulcao C, Ferreira SR, Giuffrida FM, Ribeiro-Filho FF. The new adipose tissue and adipocytokines. Curr Diabetes Rev 2006; 2: 19-28.

2. Fantuzzi G. Adipose tissue, adipokines and inflammation. J Allergy Clin Immunol 2006; 115: 911-919.

3. Chang, Y., T. Chang, W. Lee and L. Chuang. The relationship of visfatin/pre-b-cell colony-enhancing factor/nicotinamide phosphoribosyltransferase in adipose tissue with inflammation, insulin resistance, and plasma lipids. Metabolism 2010; 59 (1): 93-99.

4. Guzik TJ, Mangalat D, Korbut R. Adipocytokines - novel link between inflammation and vascular function? J Physiol Pharmacol 2006; 57: 505-28.

5. Sethi JK, Vidal-Puig A. Visfatin: the missing link between intra-abdominal obesity and diabetes? Trends Mol Med 2005; 11: 344-7.

6. FukuharaA, MatsudaM, NishizawaM, et al. Visfatin: aprotein secretedby visceral fat that mimics the effects of insulin. Science 2004; 21: 426-30.

7. Boucher J, Masri B, Apelin. A newly identified adipokine upregulated by insulin and obesity. Endocrinology, 2005; 146: 1764-1771.

8. Fukuhara A, Matsuda M, Nishizawa M, et al. Visfatin: a protein secreted by visceral fat that mimics the effects of insulin. Science 2005; 307: 426-430.

9. Adeghate, E. Visfatin: Structure, function and relation to diabetes mellitus and other dysfunctions. Curr Med Chem 2008; 15(18): 1851-1862. 
10. Haider DG, Schindler K, Schaller G et al. Increased plasma visfatin concentrations in morbidly obese subjects are reduced after gastric banding. J Clin Endocrinol Metab 2006; 91: $1578-81$

11. Sandeep, S., K. Velmurugan and R. Deepa. Serum visfatin in relation to visceral fat, obesity and type 2 diabetes mellitus in Asian Indian. Metabolism Clinical and Experimental 2006; 55: 565- 570.

12. Shaheena Naz1, Muniza Saeed 2, Qamar Shakoor Sandhu1, et al. Serum Levels of Visfatin and Interleukin-6 in Non-Obese Versus Obese Men with Coronary Artery Disease. Journal of the College of Physicians and Surgeons Pakistan 2017; 27(2): 71-74 71

13. Saboor Aftab SA, Reddy N, Smith E, et al. Obesity and Type 2 Diabetes Mellitus Intern Med Open Access 2014; S6: 002.

14. World Health Organization. Diabetes, Fact sheet $\mathrm{N}^{\circ} 312$ Updated March 2013.

15. Mahmood N, Jamal Q, Junejo AM. The relationship of visfatin with measures of obesity in patients of diabetic nephropathy. Pak J Med Sci 2010; 26(3): 556-561

16. Fahmida Kabir, FM Khan, Moinuddin Ahmed. Association of plasma visfatin with diabetes subjects Journal of Medical College for women and hospital 2011; 9(2): 53-57

16. Nishida C. Appropriate body mass index for Asian populations and its implications for policy and intervention strategies. Lancet 2004; 363: 157-163.
17. Esteghamati A, Alamdari A, Zandieh A, et al. Serum visfatin is associated with type 2 diabetes mellitus independent of insulin resistance and obesity. Diabetes Res Clin Pract 2011; 91: 154-158.

18. Pagano C, Pilon C, Olivieri M, et al. Reduced plasma visfatin/pre B-cell colony enhancing factor in obesity is not related to insulin resistance in humans. J Clin Endocrinol Metab 2006; 91: 3165-70.

19. Samal B, Sun Y, Stearns G, Xie C, Suggs S, et al. Cloning and characterization of the cDNA encoding a novel human pre-B-cell colonyenhancing factor. Mol Cell Biol 1994; 14(2): 1431-1437.

20. Chen MP, Chung FM, Chang DM, et al. Elevated plasma level of visfatin/pre-B cell colonyenhancing factor in patients with type 2 diabetes. $\mathrm{J}$ Clin Endocrine Metab 2006; 91: 295-9.

21. Jin $\mathrm{H}$, Jiang $\mathrm{B}$, Tang $\mathrm{J}$ et al. Serum visfatin concentrations in obese adolescents and its correlation with age and high-density lipoprotein cholesterol. Diabetes Res Clin Pract. 2008; 79(3): 412-8.

22. Kolsgaard ML, Wangensteen $\mathrm{T}$, Brunborg C, et $a l$. Elevated visfatin levels in overweight and obese children and adolescents with metabolic syndrome. Scand J Clin Lab Invest 2009; 69: 858-864.

23. Brendt $\mathrm{J}$, Kling $\mathrm{N}$, Kralishe $\mathrm{S}$ et al.: Plasma visfatin concentrations and fat depot-specific mRNA expression in humans. Diabetes 2005; 54: $2911-2916$ 